\title{
Evidence of quantum size effect in nanocrystalline silicon by optical absorption
}

\author{
Takahiro Matsumoto, ${ }^{1}$ Jun-ichi Suzuki, ${ }^{2}$ Masato Ohnuma, ${ }^{3}$ Yoshihiko Kanemitsu, ${ }^{4}$ and Yasuaki Masumoto ${ }^{1,5}$ \\ ${ }^{1}$ Single Quantum Dot Project, ERATO, Japan Science and Technology Corporation, 5-9-9 Tokodai, Tsukuba 300-2635, Japan \\ ${ }^{2}$ Advanced Science Research Center, Japan Atomic Energy Research Institute, Tokai, Ibaraki 319-1195, Japan \\ ${ }^{3}$ National Research Institute for Metals, 1-2-1 Sengen, Tsukuba 305-0047, Japan \\ ${ }^{4}$ Graduate School of Materials Science, Nara Institute of Science and Technology, Ikoma, Nara 630-0101, Japan \\ ${ }^{5}$ Institute of Physics, University of Tsukuba, Tsukuba 305-8571, Japan
}

(Received 30 October 2000; published 30 April 2001)

\begin{abstract}
The optical absorption spectrum in nanocrystalline silicon $(n-\mathrm{Si})$ was determined from both light transmittance and reflectance measurements. We observed that $n$-Si has a phonon structure in the optical absorption spectrum. This structure originates from momentum-conserving TO phonon absorption and emission, and provides direct evidence that $n$-Si is an indirect-band-gap semiconductor with quantum size effects. By using small-angle $\mathrm{x}$-ray scattering to measure the nanocrystal size distribution, we found that the band-gap widening varies as $(1 / L)^{1.6}$ with decreasing nanocrystal diameter $L$.
\end{abstract}

DOI: 10.1103/PhysRevB.63.195322

PACS number(s): 78.67.-n, 61.10.Eq, 61.46.+w,

To integrate optical technology with current silicon electronics technology, over the last ten years quantum size effects in silicon nanocrystals have been extensively studied. ${ }^{1-3}$ Many studies have been done to clarify the quantum size effects from the point of view of structural characterization and optical techniques ${ }^{4,5}$ such as transmission electron microscopy, ${ }^{6}$ size-selective precipitation and sizeexclusion chromatography, ${ }^{7}$ x-ray absorption measurements, ${ }^{8}$ and resonantly excited photoluminescence (PL) measurements. ${ }^{9,10}$ In particular, the effect of size of nanocrystals on the band-gap widening is of central importance for understanding the confinement effect. Band-gap widening has therefore been studied by analyzing the PL spectrum in nanocrystalline $\mathrm{Si},(n-\mathrm{Si})$. However, PL originates not only from the quantum confinement effect but also from defects, impurities, and surface chemistry. Thus, the study of PL cannot give a clear solution to the problem of quantum size effects in $n$-Si.

By doing both transmission and reflection measurements to determine the optical absorption spectrum of $n-\mathrm{Si},{ }^{11}$ we show in this work that $n$-Si made using electrochemical anodization $^{12}$ has a step structure and a blueshift in the absorption edge of the optical absorption spectrum. This step structure originates from momentum-conserving transverse optical (TO) phonon absorption and emission, and provides direct evidence that $n$-Si is affected by quantum size effects and has the nature of an indirect-band-gap semiconductor. By using the step structure in the optical absorption spectrum, even without knowing parameters such as the porosity and the thickness of the film, we can accurately determine the band gap of $n$-Si samples with inhomogeneous size distributions.

By using small-angle $\mathrm{x}$-ray scattering (SAXS) to measure nanocrystal size distributions, we show that the magnitude of the band-gap widening varies as $(1 / L)^{1.6}$ with decreasing nanocrystal diameter $L$. This exponent coincides with the results of theoretical calculations with Coulomb interactions. $^{13,14}$

Free-standing $n$-Si films with porosity from $60 \%$ to $90 \%$ were formed by anodization of (100)-oriented $p$-type silicon wafers with 1-10 $\Omega \mathrm{cm}$ resistivity in a $\mathrm{HF}$-ethanol solution at constant current density of $20 \mathrm{~mA} / \mathrm{cm}^{2}$ for $90-105 \mathrm{~min}$. The HF concentrations, between $20 \%$ and $35 \%$, were adjusted to obtain the desired porosity. The films were then detached from the substrates by electropolishing at a current density of $700 \mathrm{~mA} / \mathrm{cm}^{2}$. These free-standing films were dried by a supercritical drying process using pentane as the drying liquid to obtain thick and high-porosity layers. ${ }^{15,16}$ The thickness of these films is in the range of 90 to $100 \mu \mathrm{m}$. The porosities of the samples were determined gravimetrically by measuring their weight and volume.

In order to determine the size of $n$-Si crystals SAXS measurements were carried out for each sample using a pinhole-collimated instrument, Rigaku-PSAXS-3S, with a $\mathrm{Cu}$ target. Incident $\mathrm{x}$ rays with the wavelength of $\mathrm{Cu} K \alpha$ radiation $(\lambda=0.154 \mathrm{~nm})$ were injected perpendicularly to the film plane, giving the structural information along the film plane. Scattered $x$ rays were detected by a one-dimensional position-sensitive detector placed at 0.5 and $1 \mathrm{~m}$ from the sample.

Transmission $(T)$ and reflection $(F)$ measurements were carried out at room temperature $(293 \mathrm{~K})$ using a tungsteniodine (WI) lamp and a PbS detector (P2682 of Hamamatsu Photonics Corp.) for the infrared (0.8-1.86 eV) and a photomultiplier (R928 of Hamamatsu Photonics Corp.) for the visible region $(1.85-2.5 \mathrm{eV})$. The tungsten-iodine light was dispersed by a monochromator and focused on the sample. All the scattered light both from transmission and from reflection was collected using an integrated sphere. The transmission spectrum was calibrated by measuring the transmission without the sample. The reflection spectrum was also calibrated by measuring a pressed $\mathrm{BaSO}_{4}$ powder plate as a standard material. Then the absorbance $(\alpha \delta)$ was calculated using the relation $T=(1-F)^{2} \exp (-\alpha \delta) /[1$ $\left.-F^{2} \exp (-2 \alpha \delta)\right],{ }^{11}$ where $T$ is the measured transmittance, $\delta$ is the thickness of the film, and $F$ is the reflectance.

Figure 1 shows SAXS intensities $I(q)$ obtained from $n$-Si with porosities of $p=63,79$, and $87 \%$, where the wave number $q=(4 \pi / \lambda) \sin (2 \theta / 2)$ and $2 \theta$ is a scattering angle. The scattering profiles have qualitatively similar features and 


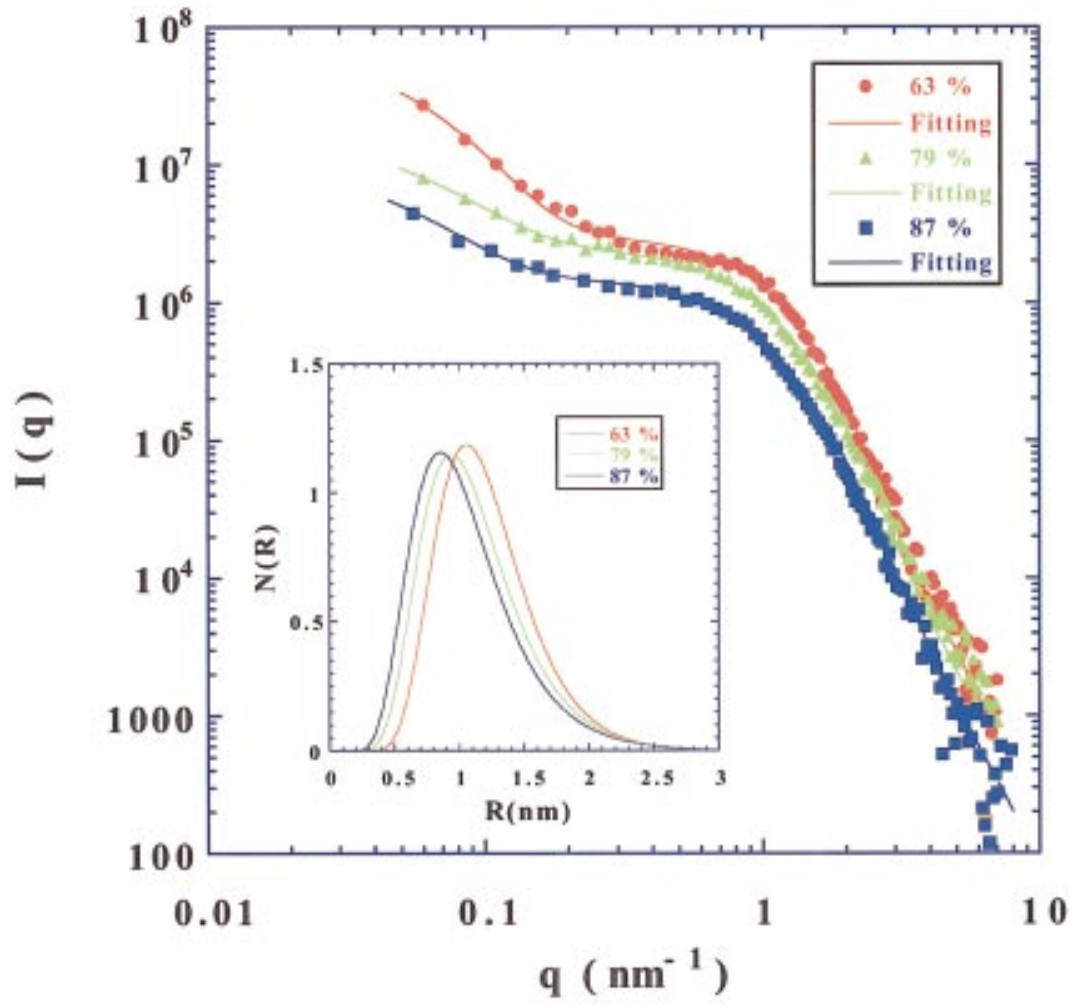

FIG. 1. (Color) SAXS intensities $I(q)$ obtained from silicon nanocrystals with the porosities of $p=63,79$, and $87 \%$, where $q$ $=(4 \pi / \lambda) \sin (2 \theta / 2) \quad(2 \theta$ is the scattering angle $)$. The inset shows the size distribution of nanocrystalline silicon with porosities of $p=63,79$, and $87 \%$.

they can be divided into two regions on the $q$ axis characterized by clear humps around $q=1 \mathrm{~nm}^{-1}$. In the higher- $q$ region, the scattering intensities are proportional to $q^{-4}$, the so-called Porod law. ${ }^{17}$ This well-known $q$ dependence implies that the interface between the nanocrystal and the pore shows a sharp boundary like a step function. Furthermore, we observed a size dependence of the scattering intensity. Reduction of the scattering intensity in the whole $q$ region shows a decrease of the scattering volume fraction, indicating a decrease of the nanocrystal diameter. This is because our small-angle neutron scattering experiments show that the scattering signal originates from nanocrystal cores, and that the number of nanocrystals per unit volume is almost the same for all porosities, on the order of $1 \times 10^{19} \mathrm{~cm}^{-3}$. In the lower- $q$ region, the scattering intensities gradually increase toward $q=0 \mathrm{~nm}^{-1}$ and the gradient of the scattering intensity decreases as the porosity increases. This result indicates that a small number (about $0.5 \%$ ) of the nanocystals aggregate with each other and that the number of aggregated nanocrystals decreases as the porosity increases.

According to these results, we quantitatively analyze the scattering intensities with a polydisperse hard spherical

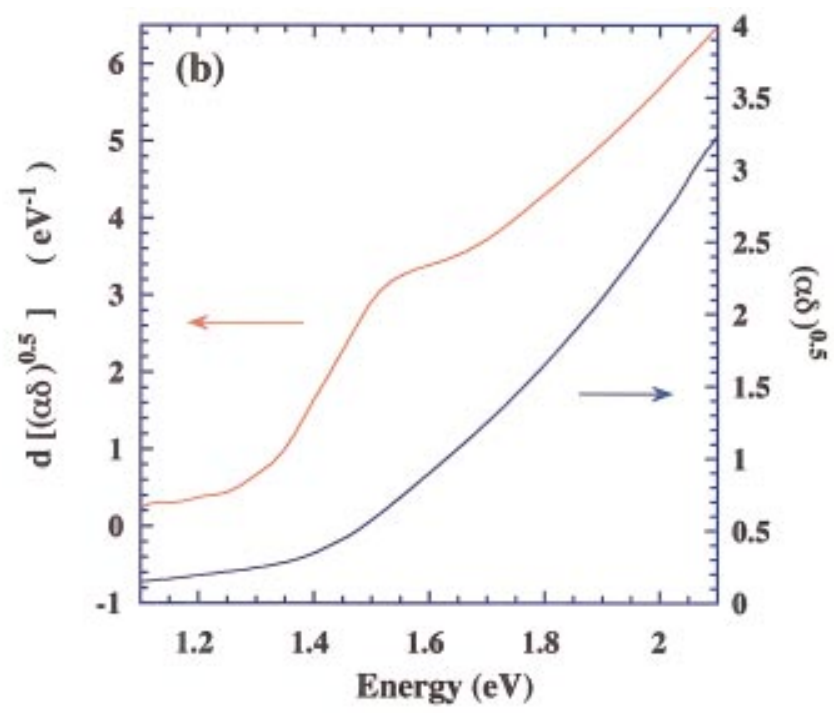

FIG. 2. (Color) The square root of the absorption coefficient $\left(\alpha^{0.5}\right)$ (solid black line) and its first derivative $\left[d\left(\alpha^{0.5}\right)\right]($ solid red line) versus photon energy for bulk Si thin film with $65 \mu \mathrm{m}$ thickness (a), and for Si nanocrystal of $2.43 \mathrm{~nm}$ diameter (b). 

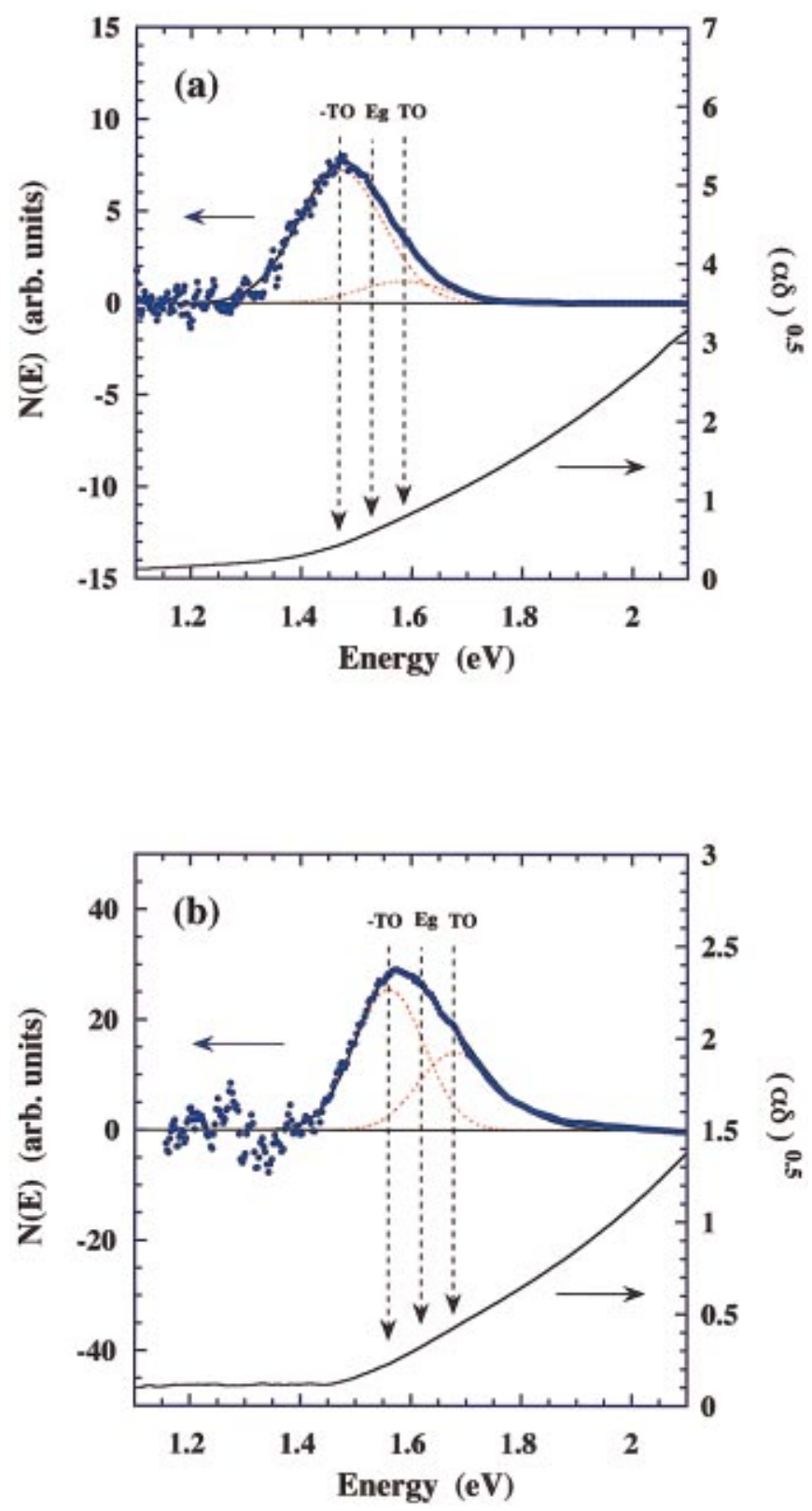

FIG. 3. (Color) Square root of the absorption coefficient $\left[(\alpha \delta)^{0.5}\right]$ (solid line), and the TO phonon peak (dots) for $2.43 \mathrm{~nm}$ Si nanocrystal (a), and for $2.24 \mathrm{~nm} \mathrm{Si} \mathrm{nanocrystal} \mathrm{(b).} \mathrm{The} \mathrm{asym-}$ metrical peak is theoretically fitted by the superposition of TO phonon absorption and emission peaks (dotted lines). Dotted arrows show the band gap $\left(E_{g}\right)$ of the nanocrystal.

nanocrystalline model. ${ }^{18}$ The scattering intensity $I(q)$ is expressed as

$$
I(q)=C_{1} \int_{0}^{\infty} \int_{0}^{\infty} S\left(q^{\prime}\right) W\left(q^{\prime}, q\right) N(R) f\left(q^{\prime}, R\right)^{2} d q^{\prime} d R
$$

where $S\left(q^{\prime}\right)$ is the structure factor for the hard spheres, $W\left(q^{\prime}, q\right)$ is the resolution function of the instrument, $N(R)$ is the normalized size distribution from a log-normal density function, $f\left(q^{\prime}, R\right)$ is the form factor of the particles with

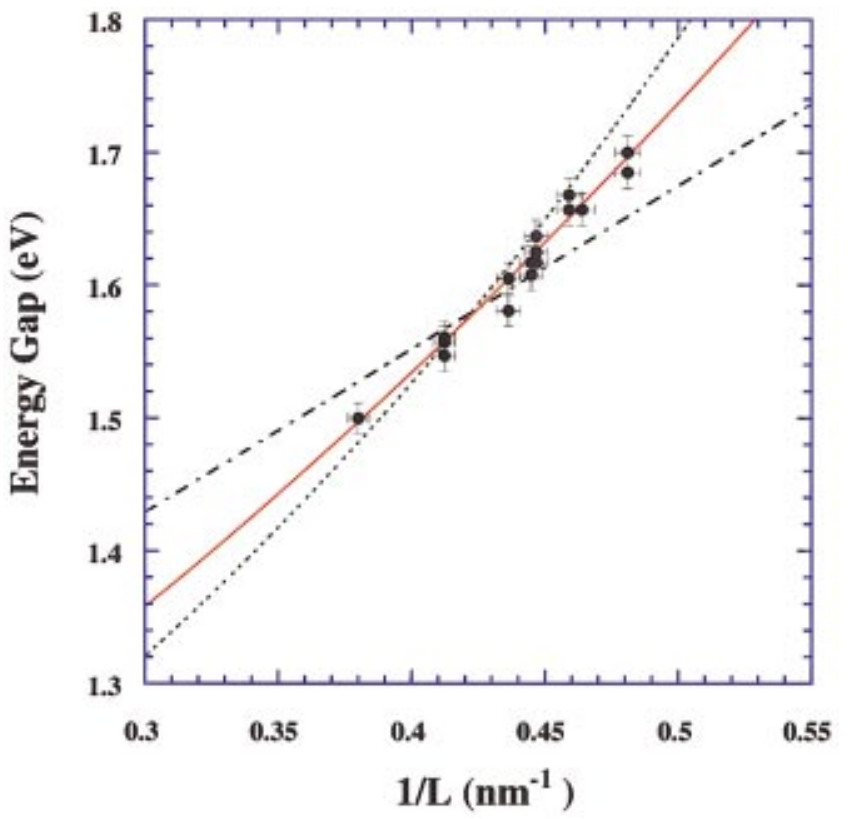

FIG. 4. (Color) Band-gap energy as a function of Si nanocrystal diameter. The band-gap upshift falls on the solid red line, $E_{g}$ $=1.06+2.05 \times(1 / L)^{1.6}$. We show both $(1 / L)^{2}$ (dotted line) and $(1 / L)$ (broken line) as a reference.

radius $R$, and $C_{1}$ is a constant parameter. ${ }^{18}$ The solid lines are the curves fitted by Eq. (1). From this fitting, we can determine the size distribution and the average radius of the film for each porosity. The inset of Fig. 1 shows the size distribution with porosities of $p=63,79$, and $87 \%$. The size distribution shifts toward the smaller radius of $n$-Si with increasing porosity. The average diameter $L$ is estimated as $2.43,2.24$, and $2.11 \mathrm{~nm}$ for porosities of $p=63,79$, and $87 \%$, respectively. The accuracy of the SAXS measurements exceeds $2 \%$, which is enough to distinguish the above diameter change. The size estimated here is smaller than the values determined by Raman scattering and transmission electron microscope analysis, ${ }^{4}$ but shows almost the same values determined by $\mathrm{Si} K$-edge $\mathrm{x}$-ray absorption measurements ${ }^{8}$ (NEXAFS) and other SAXS measurements with bimodal size distribution analysis. ${ }^{19}$

Figures 2 show the plots of the square root of the absorption coefficient (solid black line) and its first derivative (solid red line) versus photon energy for a bulk Si thin film with 65 $\mu \mathrm{m}$ thickness (a) and for a $n$-Si film (b). The $(\alpha)^{0.5}$ curve of $c$-Si exhibits two segments in the vicinity of the fundamental band gap around $1.12 \mathrm{eV}$. The first derivative of these steps in particular clearly shows two sharp peaks at the lower- and higher-energy sides of the band gap as drawn by the dotted arrows. These two contributions are due to absorption and emission of momentum-conserving TO phonons with the energy of $57 \mathrm{meV} .{ }^{20}$ Both the linear dependence and the two TO phonon peaks in the derivative of the $(\alpha)^{0.5}$ curve give evidence for Si being an indirect-band-gap semiconductor.

The $(\alpha \delta)^{0.5}$ curve of $n$-Si with $2.43 \mathrm{~nm}$ diameter clearly shows both a band-gap upshift from 1.12 to around $1.4 \mathrm{eV}$ and a linear dependency on photon energy. In addition, it is possible to observe a step near the absorption edge. The de- 
rivative of this curve shows a clear peak with a full width at half maximum (FWHM) of $200 \mathrm{meV}$ as drawn by the solid red line. The appearance of this peak in the derivative is likely to originate from momentum-conserving TO phonon absorption and emission processes of $n$-Si with inhomogeneous size broadening.

Figure 3(a) and 3(b) show the square root of the absorbance (solid line), and the peak extracted from the derivative of the $(\alpha \delta)^{0.5}$ curve for $2.43 \mathrm{~nm} n$-Si (a) and $2.24 \mathrm{~nm} n$-Si (b). From these figures, we can clearly observe a higherenergy shift of the peak with decreasing nanocrystal size, together with asymmetrical broadening at the higher-energy side. Both the higher-energy shift and the asymmetrical spectrum suggest that the peak originates from the superposition of inhomogeneously broadened two-TO-phonon peaks.

Here, we shall theoretically draw the peak spectrum with two TO phonon curves. The absorption of an indirect-bandgap semiconductor with nanostructural size distribution can be described as ${ }^{10}$

$$
\begin{aligned}
\alpha= & \sum_{n=-m}^{n=+m} \nu_{n}\left(N_{P}+\frac{1}{2} \pm \frac{1}{2}\right)^{|n|} \int_{E_{s}}^{E_{l}} \theta(\hbar \omega-n \hbar \Omega-E) \\
& \times(\hbar \omega-n \hbar \Omega-E)^{2} D\left(E-E_{g}\right) d E,
\end{aligned}
$$

where $D\left(E-E_{g}\right)$ is the distribution function of $n$-Si with a center energy of $E_{g}, \hbar \Omega$ is the TO phonon energy, $\theta$ is the Heaviside step function, $N_{p}=[\exp (\hbar \Omega / k T)-1]^{-1}$ is the Bose factor, the subscript $n>0$ indicates phonon emission and $n$ $<0$ phonon absorption, $\nu_{n}$ represents the intensity of each phonon structure, and the size inhomogeneity is integrated from the smallest size $E_{s}$ to the largest size $E_{l}$. Near the band gap of the nanocrystal, one-phonon absorption and onephonon emission dominate the absorption process; therefore, we consider $n=+1$ and $n=-1$ for the above equation. By combining the experimentally observed absorption coefficient $\alpha$ with Eq. (2), we fit the size-dependent peaks shown in Fig. 3. Black solid lines are theoretically fitted curves. These curves are composed of the sum of the same two TO phonon curves (absorption and emission) with the energy separation of $2 \hbar \Omega$. This result clearly shows that the peak deduced from the absorption spectrum shifts to higher energy with decreasing size of the nanocrystal and that it can be fitted by an inhomogeneously broadened TO phonon absorption and emission spectrum. Even though the oscillator strength as a function of size remains unknown, by taking the middle energy between these two TO phonon peaks, we can accurately determine the band gap $E_{g}$ as a function of the average diameter of this inhomogeneously broadened system. The band gap $E_{g}$ thus determined is $1.527 \mathrm{eV}$ for the $2.43 \mathrm{~nm}$ nanocrystal [Fig. 3(a)], and $1.617 \mathrm{eV}$ for the 2.24 nm nanocrystal [Fig. 3(b)].
Both the linear dependence of the $(\alpha \delta)^{0.5}$ curve and the TO phonon step in the vicinity of the blueshifted absorption edge clearly provide direct evidence that $n$-Si is affected by quantum size effects and has the nature of an indirect-badgap semiconductor.

Figure 4 shows the band-gap energy as a function of the average diameter of $n$-Si determined by the SAXS measurements. The band gap shifts to higher energy with decreasing crystal size. Determination by the above analysis shows that the magnitude of the band-gap widening for 2.5 $\mathrm{nm} n$ - $\mathrm{Si}$ is about $400 \mathrm{meV}$. This value for the size is smaller than the previously reported values determined by PL excitation spectrum (PLE) measurements and PL peak measurements. ${ }^{4,5,8}$ We believe that the band gap determined here using TO phonon steps gives the correct value as compared to PL and PLE measurements, because the PL process is affected not only by direct absorption but also by successive relaxation processes involving defects, impurities, and surface chemistry.

The band-gap energies determined here fall on the fitted line $E_{g}=1.06+2.05 \times(1 / L)^{1.6}$ as shown by the solid red line in Fig. 4. The exponent 1.6 determined by least-squares fitting is smaller than 2 (dotted line), which is expected from the effective-mass approximation, but is larger than 1 (broken line) determined by Schuppler et al. ${ }^{8}$ The experimentally deduced exponent 1.6 is almost the same as that expected from a third-nearest-neighbor nonorthogonal-basis tightbinding calculation ${ }^{14}$ (TNNTB) or empirical pseudopotential plane-wave (EPPW) theory with Coulomb and correlation energy corrections. ${ }^{13}$ However, the discrepancy of the bandgap energy (the discrepancy of the coefficient 2.05) is still large, on the order of $200 \mathrm{meV}$. This discrepancy seems to originate from several points. First, the band-gap calculations were based on an ideal model, whereas the $n$-Si samples were different in shape and configuration of the surface termination. Second, the theories referred to here do not include the size dependence of the dielectric constant although this constant is greatly affected by the nanocrystal size. ${ }^{21,22}$ Thus, agreement between the experimental results and the theoretical analysis could be obtained by including the above points.

Here we find that silicon nanocrystals have a step structure originating from TO phonon absorption and emission. This step structure provides direct evidence that the silicon nanocrystal is affected by quantum size effects and has the nature of an indirect-band-gap semiconductor. The quantitative method introduced here offers an accurate determination of the band gap of this inhomogeneous system. By using SAXS to measure the nanocrystal size distribution, we show that the band-gap upshift is proportional to $(1 / L)^{1.6}$, which exponent agrees well with TNNTB or EPPW calculations.
${ }^{1}$ S. S. Iyer and Y.-H. Xie, Science 260, 40 (1993).

${ }^{2}$ Z. H. Lu, D. J. Lockwood, and J.-M. Baribeau, Nature (London) 378, 258 (1995).

${ }^{3}$ D. Lockwood, Light Emission in Silicon: From Physics to
Devices (Academic, San Diego, 1998).

${ }^{4}$ Y. Kanemitsu, Phys. Rep. 263, 15 (1995).

${ }^{5}$ A. G. Cullis, L. T. Canham, and P. D. J. Calcott, J. Appl. Phys. 82, 909 (1997). 
${ }^{6}$ A. G. Cullis and L. T. Canham, Nature (London) 353, 335 (1991).

${ }^{7}$ W. L. Wilson, P. F. Szajowski, and L. E. Brus, Science 262, 1242 (1993).

${ }^{8}$ S. Schuppler et al., Phys. Rev. Lett. 72, 2648 (1994).

${ }^{9}$ P. D. J. Calcott et al., J. Lumin. 57, 257 (1993).

${ }^{10}$ T. Suemoto et al., Phys. Rev. Lett. 70, 3659 (1993).

${ }^{11}$ J. I. Pankov, Optical Processes in Semiconductors (Dover, New York, 1971), p. 93.

${ }^{12}$ L. T. Canham, Appl. Phys. Lett. 57, 1096 (1990).

${ }^{13}$ A. Zunger and L.-W. Wang, Appl. Surf. Sci. 102, 350 (1996).

${ }^{14}$ J. P. Proot, C. Delerue, and G. Allan, Appl. Phys. Lett. 61, 1948
(1992).

${ }^{15}$ L. T. Canham et al., Nature (London) 368, 133 (1994).

${ }^{16}$ D. Bellet and G. Dolino, Phys. Rev. B 50, 17162 (1994).

${ }^{17}$ G. Porod, Kolloid-Z. 124, 83 (1951).

${ }^{18}$ G. Porod, in Small-Angle X-Ray Scattering, edited by O. Glatter and O. Kratky (Academic, London, 1982), p. 17.

${ }^{19}$ M. Binder et al., Thin Solid Films 276, 65 (1996).

${ }^{20}$ G. G. Macfarlane et al., J. Phys. Chem. Solids 8, 388 (1959).

${ }^{21}$ L.-W. Wang and A. Zunger, Phys. Rev. Lett. 73, 1039 (1994).

${ }^{22}$ M. Lannoo, C. Delerue, and G. Allan, Phys. Rev. Lett. 74, 3415 (1995). 